\title{
ACMG policy statement: updated recommendations regarding analysis and reporting of secondary findings in clinical genome-scale sequencing
}

\author{
ACMG Board of Directors ${ }^{1}$
}

\begin{abstract}
Disclaimer: These recommendations are designed primarily as an educational resource for medical geneticists and other health-care providers to help them provide quality medical genetics services. Adherence to these recommendations does not necessarily ensure a successful medical outcome. These recommendations should not be considered inclusive of all proper procedures and tests or exclusive of other procedures and tests that are reasonably directed to obtaining the same results. In determining the propriety of any specific procedure or test, geneticists and other clinicians should apply their own professional judgment to the specific clinical circumstances presented by the individual patient or specimen. It may be prudent, however, to document in the patient's record the rationale for any significant deviation from these recommendations.
\end{abstract}

As genome-scale sequencing is increasingly applied in clinical medicine, complex issues arise regarding the extent to which primary data should be analyzed and reported. At the present time, the most common clinical application of massively parallel sequencing lies in its use as a powerful new diagnostic tool in selected patients. When such sequencing is performed, primary data files consisting of a vast number of genomic variants are generated for each individual, with that information varying greatly with regard to relevance to the specific diagnostic question. What to do with these large numbers of "secondary" or "incidental" variants (nomenclature has varied with regard to such findings; the American College of Medical Genetics and Genomics (ACMG) has now adopted "secondary findings" as standard nomenclature, as recommended by the Presidential Commission on Bioethical Issues ${ }^{1}$ ) has been a matter of considerable debate and discussion. Challenges include how extensively the primary data should be routinely analyzed and which of the many variants discovered should be reported to patients. These questions are especially challenging because although many of these variants are not clinically relevant or are uninterpretable, a minority may have important medical implications for the individual sequenced as well as for other family members.

In March 2013, the ACMG issued a set of recommendations regarding the use of clinical genome-scale sequencing. One recommendation advised laboratories performing whole-exome sequencing or whole-genome sequencing for any clinical indication to specifically analyze the sequence of 57 (later revised to 56) genes. These genes were selected based on substantial clinical evidence that pathogenic variants result in a high likelihood of severe disease that is preventable if identified before symptoms occur.

The release of this set of recommendations resulted in considerable discussion, much of it focused on whether the analysis of these 56 genes should be "mandatory" when wholeexome/whole-genome sequencing is pursued clinically, or whether patients should be able to "opt out" of such secondary analysis and reporting. This discussion was informed by a report by the Presidential Commission on Bioethical Issues regarding secondary findings and a survey administered by the ACMG to its membership in January 2014. In March 2014, the ACMG updated its recommendations, prompted in part by what appeared to be a general consensus among ACMG members and other relevant stakeholders that patients should be able to opt out of the analysis of genes unrelated to the indication for testing, and that the decision should be made during the process of informed consent before testing.

In this issue of Genetics in Medicine, we publish the results of this survey ${ }^{2}$ and articulate the current recommendations of the ACMG with regard to the analysis and return of secondary findings when clinical genome-scale analysis is pursued.

- When clinical genome-scale (e.g., whole-exome sequencing, whole-genome sequencing) sequencing is performed, written informed consent should be obtained by a qualified genetics health-care professional describing the nature of the test and addressing points such as interpretive uncertainty, privacy, possible impact on other family members, and the inevitable generation of data not immediately relevant to the clinical indication for

${ }^{1}$ American College of Medical Genetics and Genomics, ACMG Foundation for Genetic and Genomic Medicine, Bethesda, Maryland, USA. Correspondence: Michael S. Watson (mwatson@acmg.net)

ACMG Board of Directors approved these recommendations on 22 September 2014.

Submitted 23 September 2014; accepted 23 September 2014; advance online publication 13 November 2014. doi:10.1038/gim.2014.151 
sequencing. At the time of testing, the patient should be made aware that, regardless of the specific indication for testing, laboratories will routinely analyze the sequence of a set of genes deemed to be highly medically actionable so as to detect pathogenic variants that may predispose to a severe but preventable outcome.

- Patients should be informed during the consent process that, if desired, they may opt out of such analysis. However, they should also be made aware at that time of the ramifications of doing so.

- In accordance with the recent recommendations of the Presidential Commission for the Study of Bioethical Issues, as well as a lack of clear consensus in the ACMG membership survey administered in January 2014, the board recommends that the same policy should be adhered to in children as in adults; i.e., analysis of a set of selected genes to identify pathogenic variants associated with severe but preventable disease should be routinely performed. Parents should have the option during the consent process to opt out of such analysis.

- At this time, given the practical concerns and inherent difficulty of counseling patients about the features of each disorder and every gene on an ever-changing list, it is not feasible for patients to be offered the option of choosing a subset of medically actionable genes for analysis. Thus, the decision regarding routine analysis should apply to the entire set of genes deemed actionable by the ACMG.

The ACMG recognizes the complex nature of policies surrounding genome-scale testing and that positions will continue to evolve and change in response to new knowledge, new technologies, and ongoing input and discussion with our membership and the broader medical community. The ACMG will continue to explore these issues in the best interest of patients. A multidisciplinary working group has been formed to develop a process for updating and maintaining the list of genes to be routinely analyzed for secondary findings.

\section{DISCLOSURE}

The authors declare no conflict of interest.

\section{REFERENCES}

1. Presidential Commission for the Study of Bioethical Issues. Anticipate and Communicate: Ethical Management of Incidental and Secondary Findings in the Clinical, Research, and Direct-to-Consumer Contexts. http://bioethics.gov/ node/3183.

2. Scheuner MT, et al. Reporting genomic secondary findings: ACMG members weigh in Genet Med 2014;17:27-35 